\title{
Identification and staging of B-cell acute lymphoblastic leukemia using quantitative phase imaging and machine learning
}

Vinay Ayyappan ${ }^{1,+}$, Alex Chang ${ }^{1,2,+}$, Chi Zhang ${ }^{3,+}$, Santosh Kumar Paidi ${ }^{3,+}$, Rosalie Bordett ${ }^{4}$, Tiffany Liang ${ }^{4}$, Ishan Barman $3,5,6$, and Rishikesh Pandey ${ }^{4,7^{*}}$

1Johns Hopkins University, Department of Biomedical Engineering, Baltimore, MD, 21218, USA

2Johns Hopkins University, Department of Computer Science, Baltimore, MD, 21218, USA

${ }^{3}$ Johns Hopkins University, Department of Mechanical Engineering, Baltimore, MD, 21218, USA

${ }^{4}$ University of Connecticut School of Medicine, Connecticut Children's Innovation Center, Farmington, CT, 06032, USA

5 Johns Hopkins University School of Medicine, Department of Oncology, Baltimore, MD, 21287, USA.

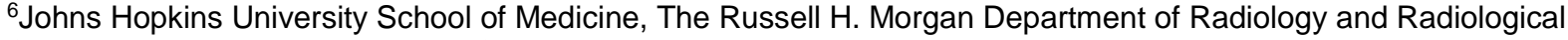

Science, Baltimore, MD, 21287, USA

${ }^{7}$ University of Connecticut, Department of Biomedical Engineering, Storrs, CT, 06269, USA

*Corresponding authors: rishikesh.pandey@uconn.edu, ibarman@jhu.edu

+These authors contributed equally to this work 


\section{Methods}

\section{Details of the pretrained network ResNet50}

We additionally applied "transfer learning" to a ResNet model, where the base layers had been pretrained ${ }^{1}$. The final layers were subsequently trained using our QPI dataset. Since ResNet is pretrained using colored images, the black and white images from our dataset were treated as 3-channel images by duplicating each image matrix and concatenating to create a tensor with depth 3 . To narrow the hyperparameter search space, a fully connected "dense" layer was appended to each pretrained model prior to our hyperparameter search. A learning rate of 0.0005 and dropout fraction of 0.5 were used. Multiple training approaches were tried, including freezing layers at progressively higher orders (i.e. until only the final layer was trained using our dataset). The Google Cloud platform was used, using the "large_model" master, worker, and parameter server types.

\section{Results}

\section{ResNet50}

We evaluated the performance of a pre-trained network, ResNet50, a large model that implements "skip connections" to jump over some layers of the network if needed during training. Although the model's size and extensive parameter sets make it a memory-intensive option, ResNet50 is compatible with transfer learning, allowing for improved initialization of the network's weights as compared to the random initialization used for a model trained from scratch ${ }^{1}$. A notable shortcoming in the ResNet's performance that we noted was its tendency (as is often true of high-capacity networks ${ }^{2}$ ) to overfit when provided with our small corpus of images, providing a class-balanced test accuracy of $38.3 \%$ (despite $\sim 76 \%$ training accuracy) in the 3-class case (Supplementary Figure S8). This is potentially attributable in part to the size discrepancy between the base image set used for pre-training (in this case, the ImageNet database, comprising tens of millions of images) and our QPI dataset used as a target ${ }^{3}$.

In analyzing the activation of neurons in the ResNet, we observed that the ResNet appeared to show very sparse activation, but strongest in response to the brightest intracellular regions (Supplementary Figure S10B). Learning heuristics used by the ResNet are difficult to identify (and likely less important given its poor classification accuracy), as they are in part a product of pretraining with a much wider palette of images than our dataset, which is distinguished by its low inter- and intra- class variability. 


\section{Supplementary Data Captions}

\section{Table Captions:}

Supplementary Table S1: Detail of the neural network architecture. Multidimensional output of the final dense layer is transformed to a scalar by the activation function, which can then be used for classification.

\section{Figure Captions:}

Supplementary Figure S1: Transport-based morphometry (TBM) analysis. The histograms of the projections of the coordinates of the images of all five classes on the most discriminant direction. The Kolmogorov-Smirnov $p$-value for the histograms separation was computed as 1.1e-5. (B) The histograms of the projections of the coordinates of the images from the four healthy donors (control class) on the most discriminant direction. The representative images correspond to the histogram coordinate, which is in units of SD of the projection. Neither the size nor the drymass were noticeably variable along the most discriminant direction.

Supplementary Figure S2: Pairwise Feature Correlations. Scatter plots showing pairwise correlations between each feature used for classification. Units are: area, px; perimeter, pixel-width; Shannon entropy (Sh), drymass (pg).

Supplementary Figure S3: Confusion matrices comparing four machine learning methods. RF, random forest; GNB, Gaussian naïve bayes; KNN, 5-nearest neighbors; QDA; quadratic discriminant analysis.

Supplementary Figure S4: Learning Curves. (A) Learning curve for the 3-class case. (B) Learning curve for the 5class case showing the relationship between classification accuracy and training-set size. Classification using traditional machine learning methods (e.g. random forest) quickly reaches optimal values even using very small sets of training data ( $\sim 50$ samples in the 3 class case, $\sim 250$ samples in the 5 -class case).

Supplementary Figure S5: Loss functions and ROC curves for the neural network. Shown are loss functions depicting the epoch-by-epoch "loss" associated with classification in the 2-class and 3-class cases. A ROC curve is also shown; the neural network was able to identify malignant cells with an AUC of 0.997

Supplementary Figure S6: Analysis of convolutional neural networks. (A) Confusion matrix comparison of random forest and convolutional network in the 3-class case. (B) Diagrams of neural networks evaluated for comparison. (C) Comparison of 3-class accuracy among different neural network architectures. (D) Histograms showing the neuron activity of the final activation layer of a 23-layer neural network (architecture (iii)) for each class.

Supplementary Figure S7: Data Augmentation. Epoch-by-epoch accuracy curves for a 23-layer network trained using image augmentation.

Supplementary Figure S8: Performance of pretrained networks. (A) Confusion matrices comparing the performance of ResNet50 against that of a 23-layer convolutional neural network. (B) Heatmaps comparing accuracy and F1 score among random forest (RF), a 23-layer convolutional network (CNN_1), a smaller convolutional network (architecture (i) from Supplementary figure S6, CNN_2), and a ResNet (ResNet50).

Supplementary Figure S9: Neurons in networks trained on QPI images show tuning for shape and color gradients. Shown are plots displaying differences in the activity of neurons in different layers of the network in response to various image archetypes designed to highlight particular image features, including size, border energy and gradient. Each subplot shows, on its x-axis, each neuron in the corresponding layer, and on the $y$-axis, the level of each neuron's activity.

Supplementary Figure S10: Analysis of pretrained and smaller convolutional network. (A) Plots displaying the activity of neurons in a 12-layer convolutional neural network in response to image archetypes highlighting size, border energy, and color gradient. (B) Activation maps and histograms describing the response of a ResNet to an example of a control and late-stage cell. 
Supplementary Table S1: Detail of the neural network architecture

\begin{tabular}{|l|l|l|l|l|}
\hline Problem-Type & Layer & Kernel Dimension & Filters & Neurons \\
\hline any & 2D Convolution & $3 \times 3 \times 1$ & 64 & \\
\hline any & Batch Normalization & & & \\
\hline any & Activation & & & \\
\hline any & Max Pooling & & & \\
\hline any & 2D Convolution & $3 \times 3 \times 1$ & 64 & \\
\hline any & Activation & & & \\
\hline any & 2D Convolution & $3 \times 3 \times 1$ & 128 & \\
\hline any & Batch Normalization & & & \\
\hline any & Activation & & & \\
\hline any & Max Pooling & & & \\
\hline any & 2D Convolution & $3 \times 3 \times 1$ & 128 & \\
\hline any & Activation & & & \\
\hline any & 2D Convolution & $3 \times 3 \times 1$ & 256 & \\
\hline any & Batch Normalization & & & \\
\hline any & Activation & & & \\
\hline any & Max Pooling & & & \\
\hline any & Global Average Pooling & & & \\
\hline any & Dense Layer & & & 1024 \\
\hline any & Dropout & & & \\
\hline any & Leaky ReLU & & & \\
\hline Binary & Sigmoid & & & \\
\hline Multiclass & Softmax & & & \\
\hline
\end{tabular}




\section{Supplementary Figure $\mathbf{S 1}$}

A
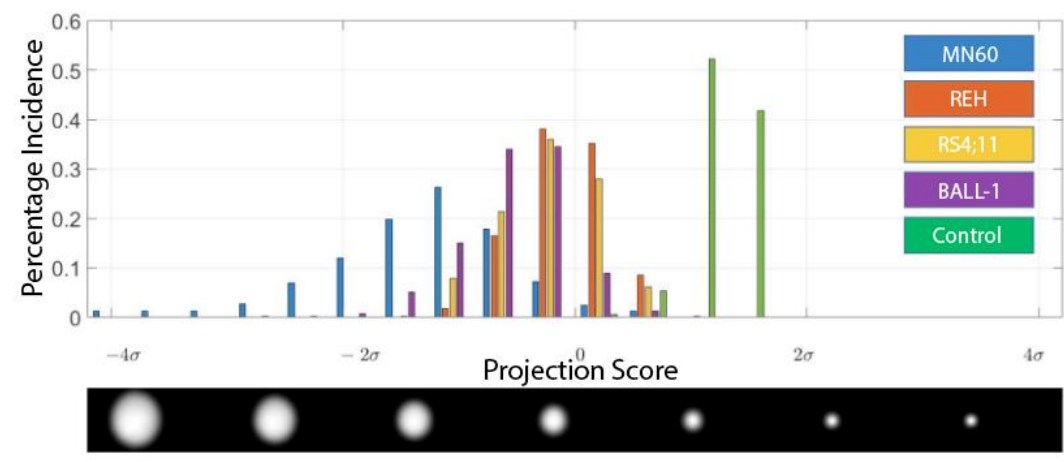

B
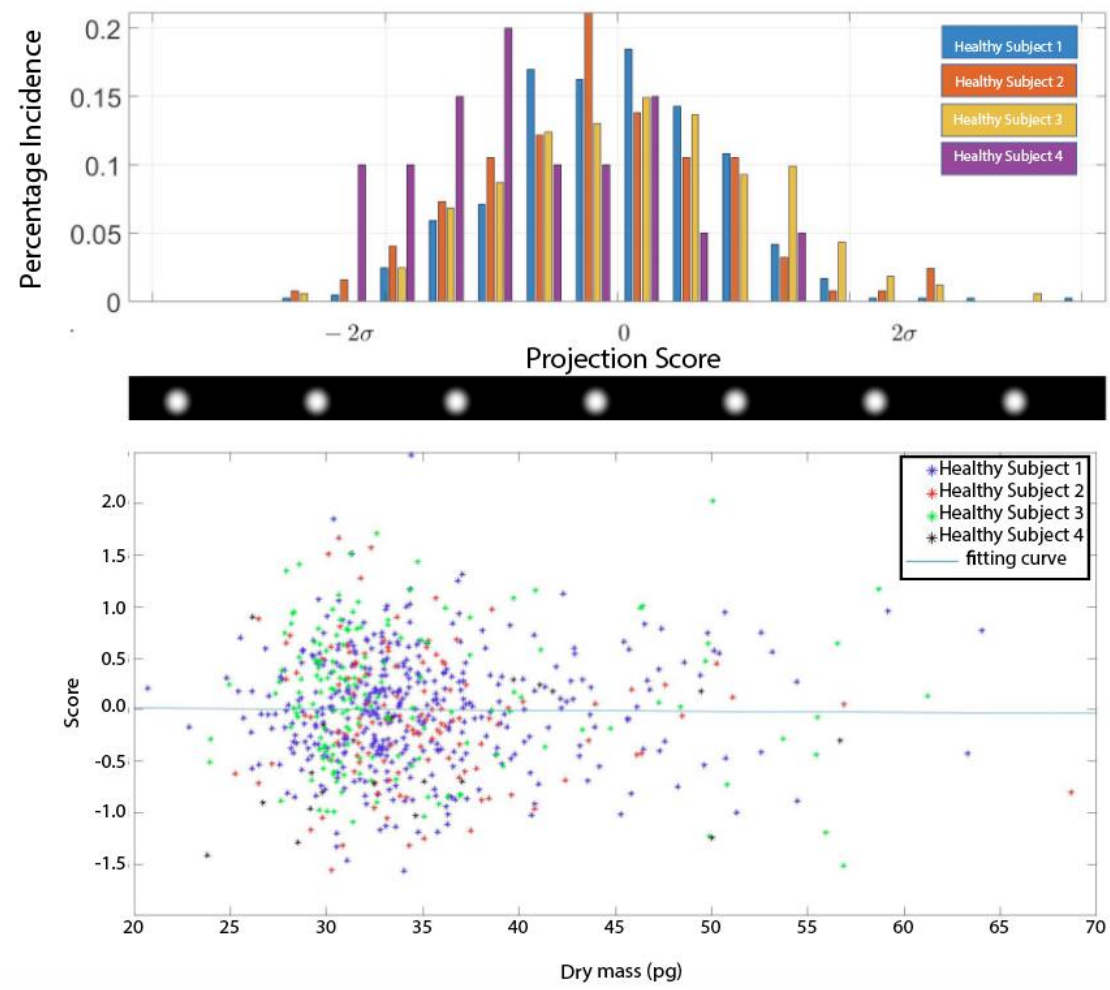


\section{Supplementary Figure S2}
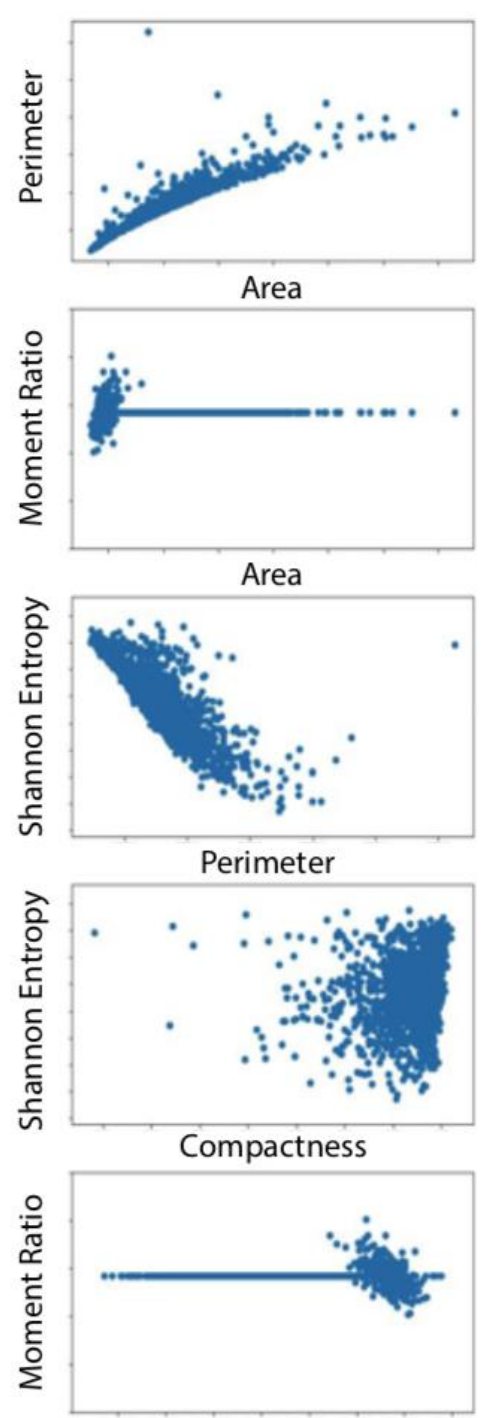

Shannon Entropy
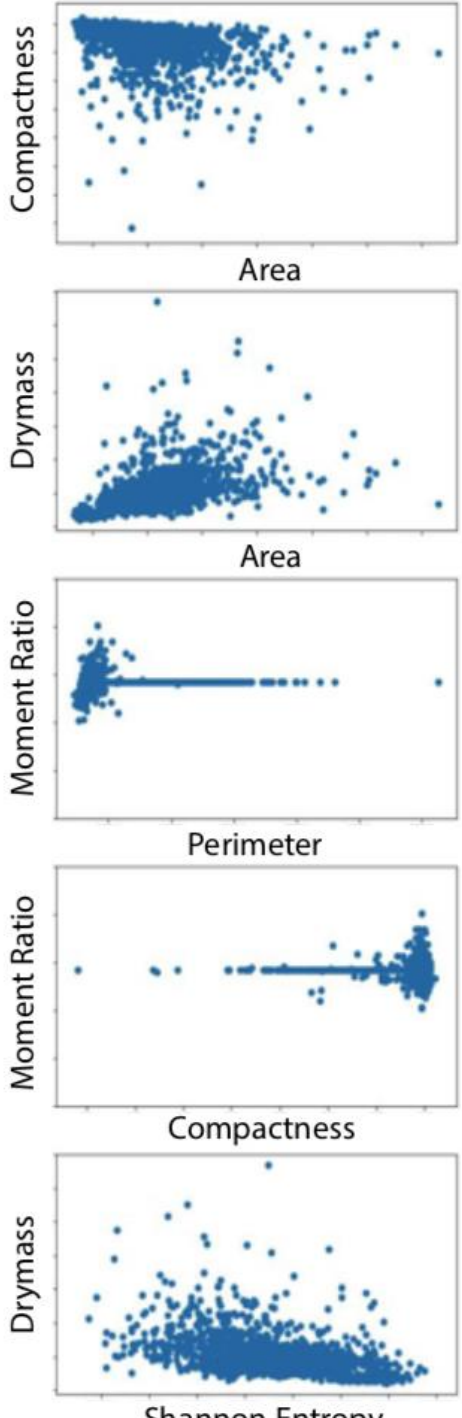

Shannon Entropy

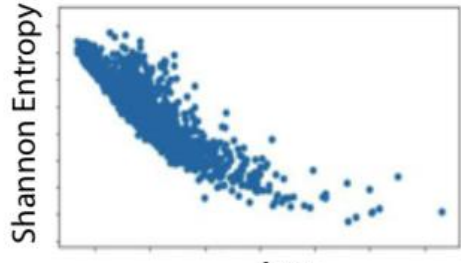

Area

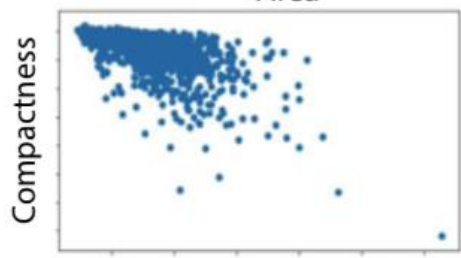

Perimeter

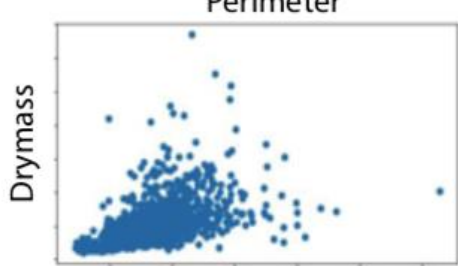

Perimeter
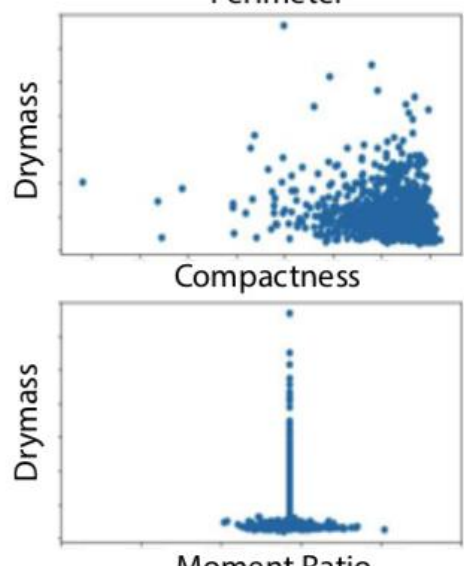


\section{Supplementary Figure S3}
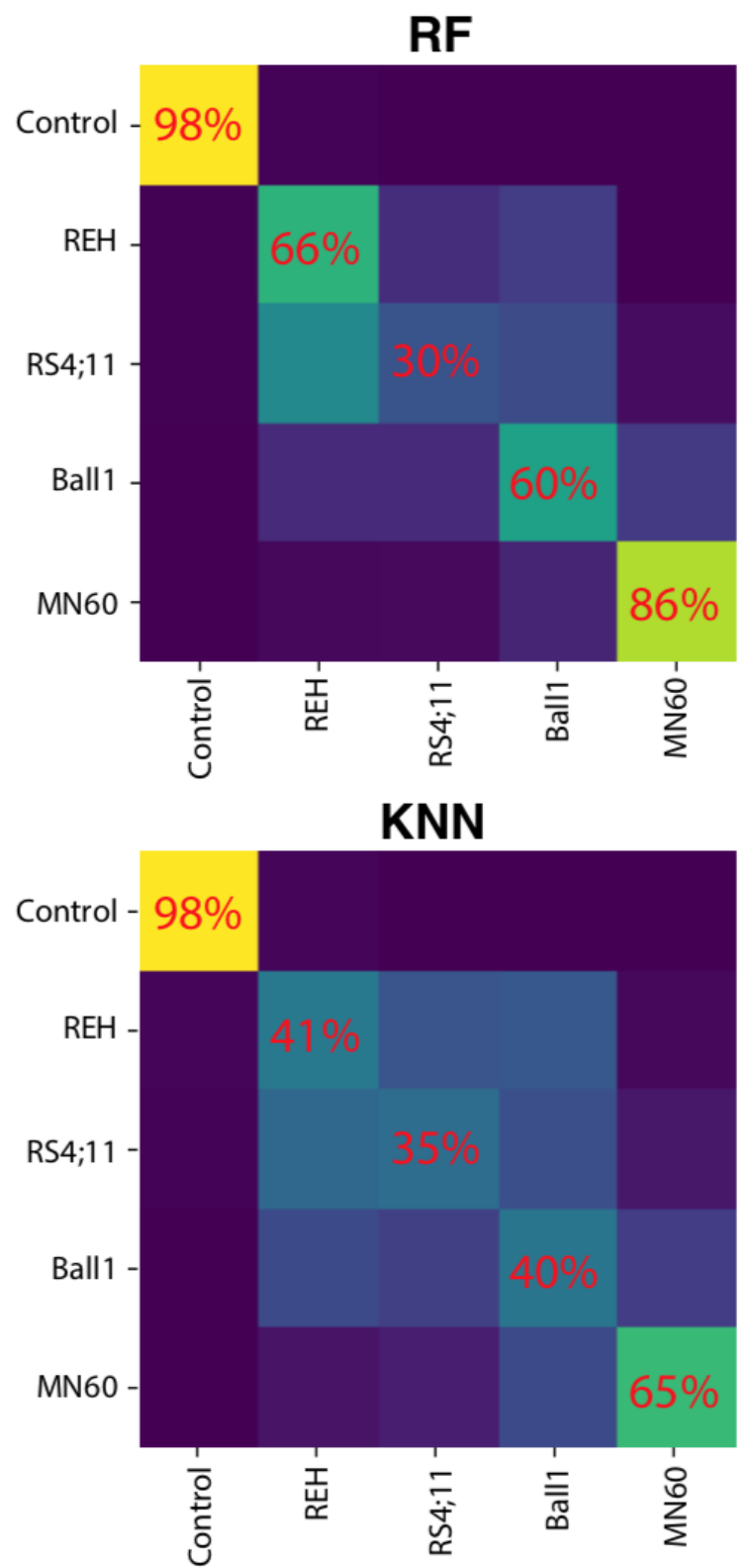

GNB

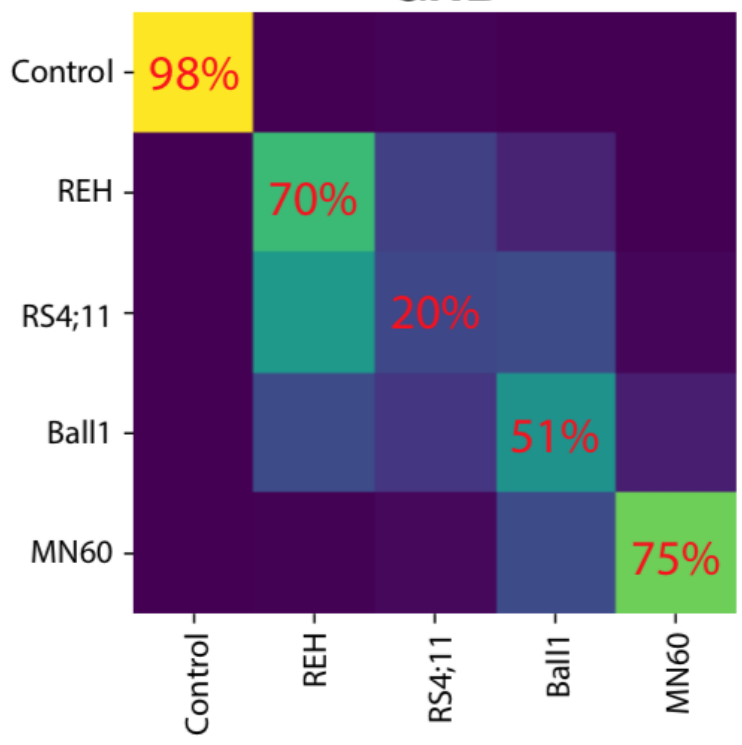

QDA

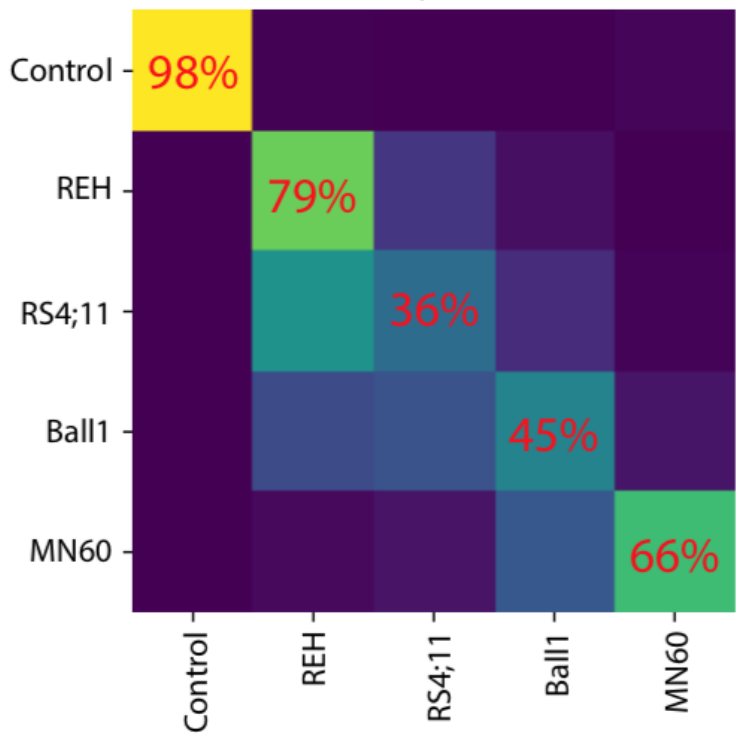




\section{Supplementary Figure S4}
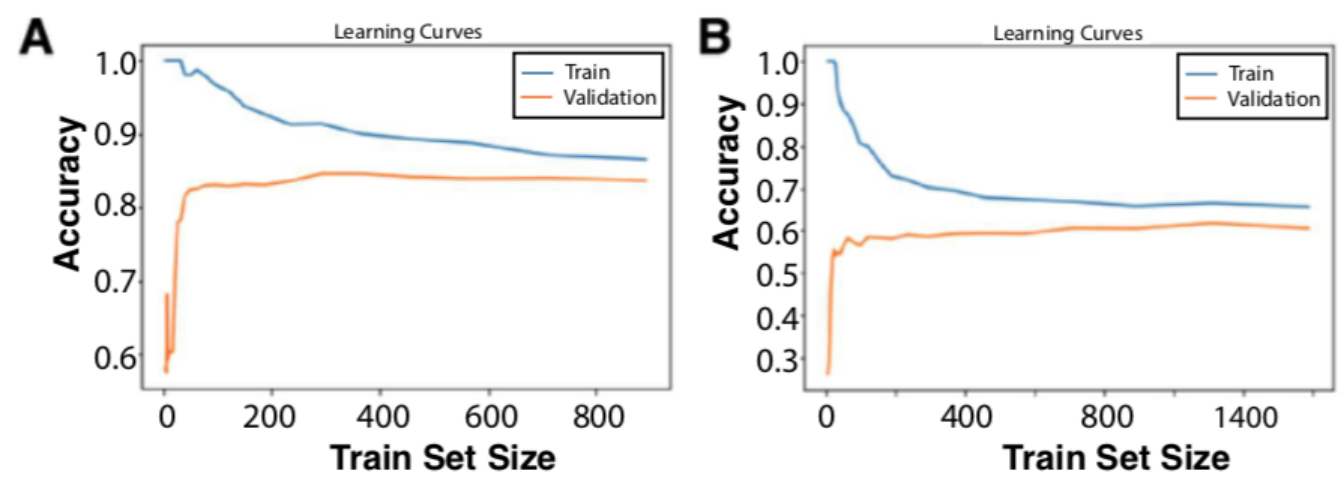


\section{Supplementary Figure S5}
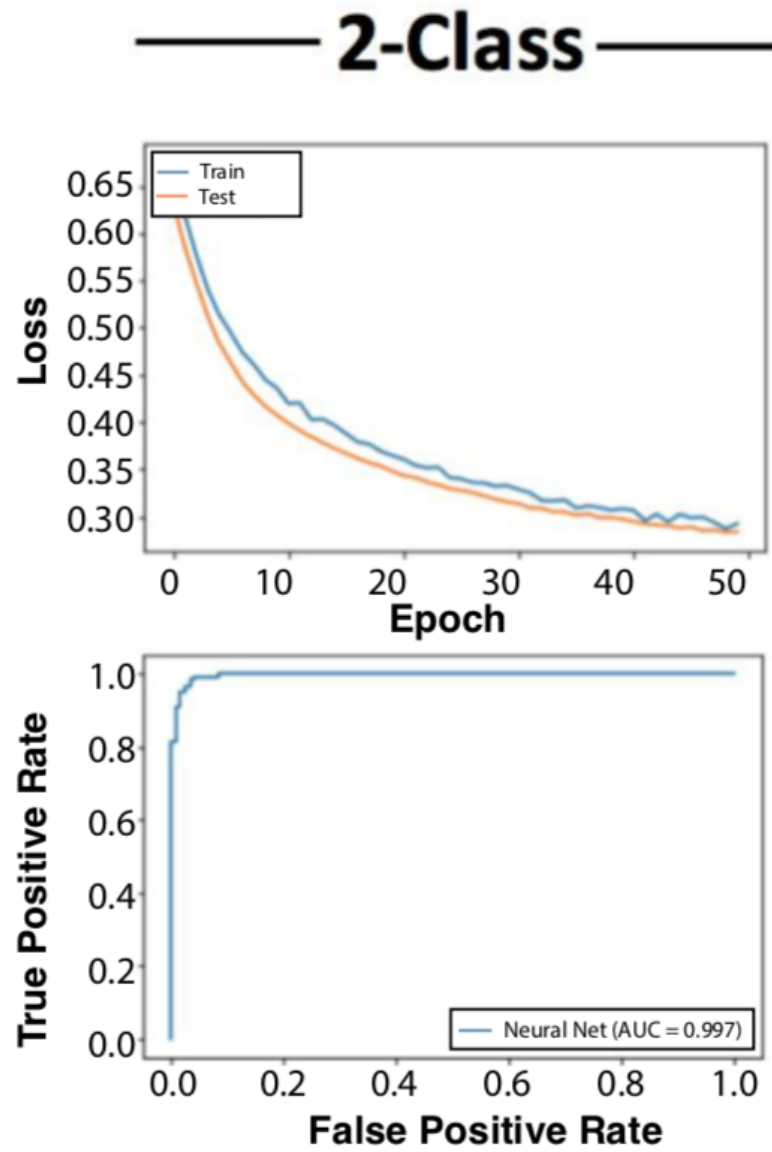

3-Class

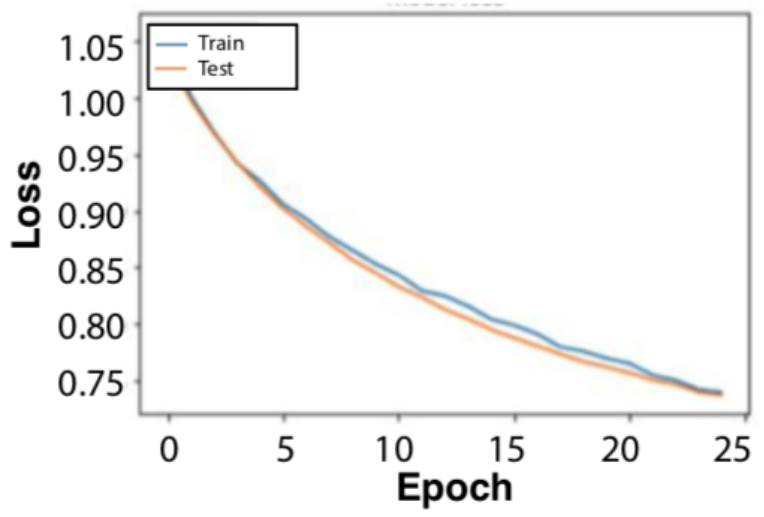




\section{Supplementary Figure S6}
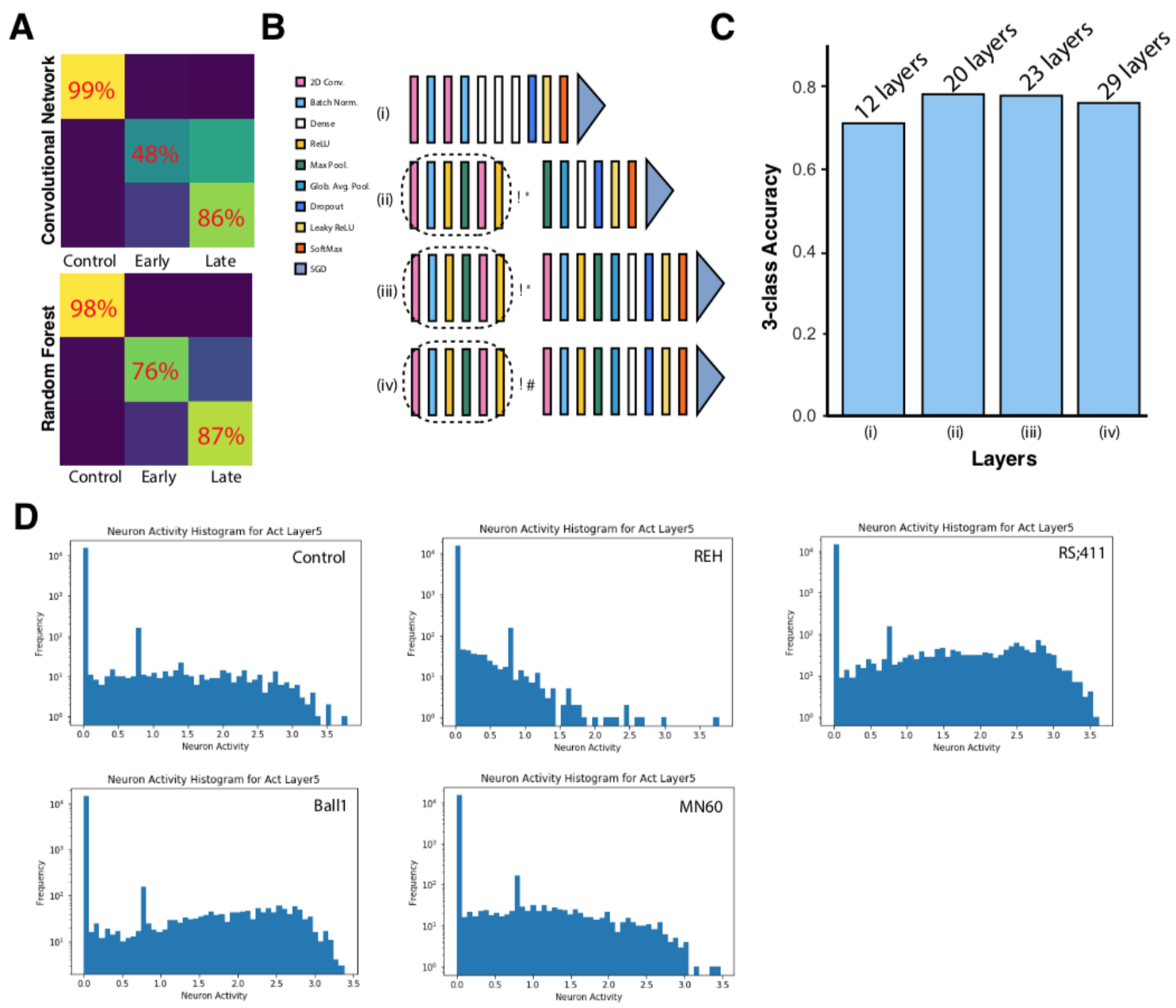


\section{Supplementary Figure S7}

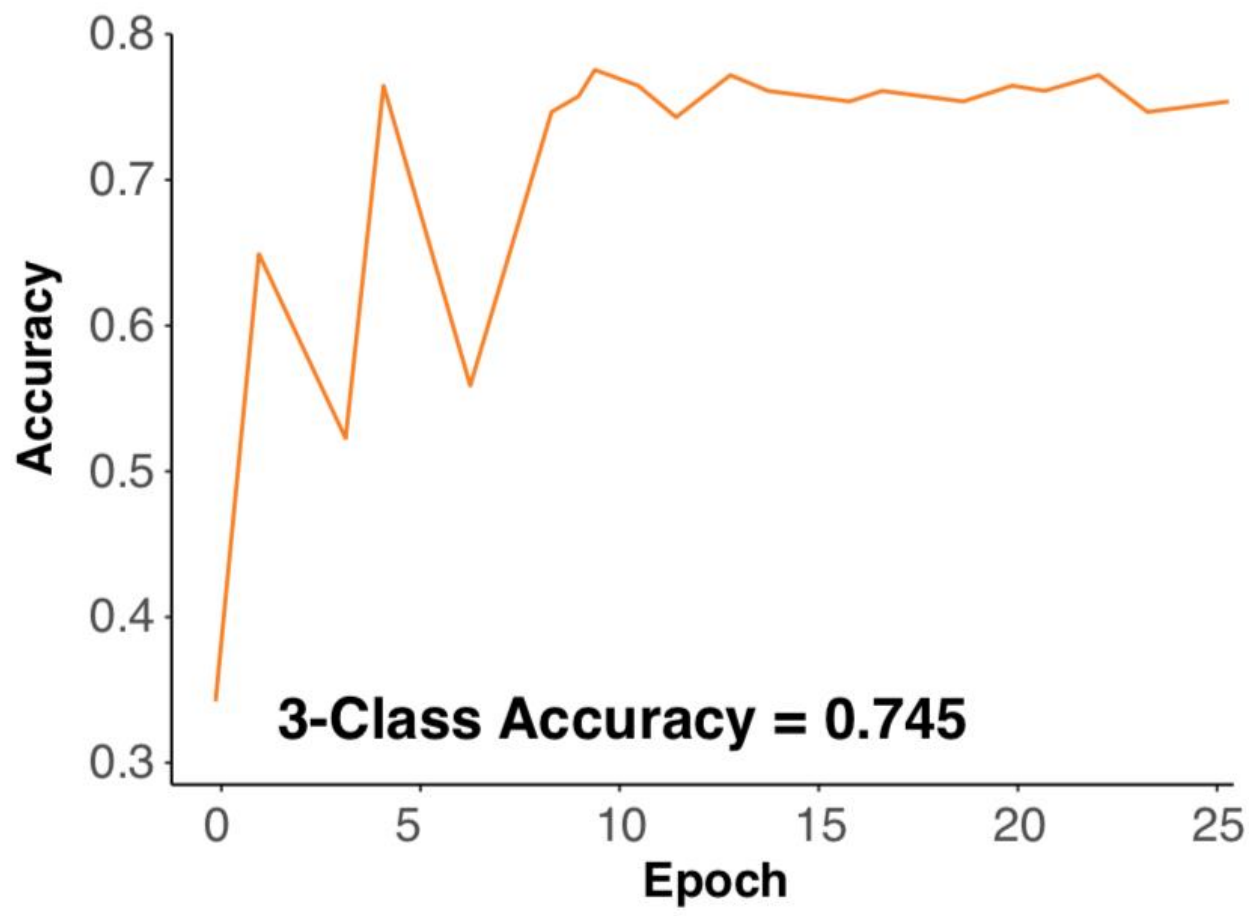




\section{Supplementary Figure S8}

A
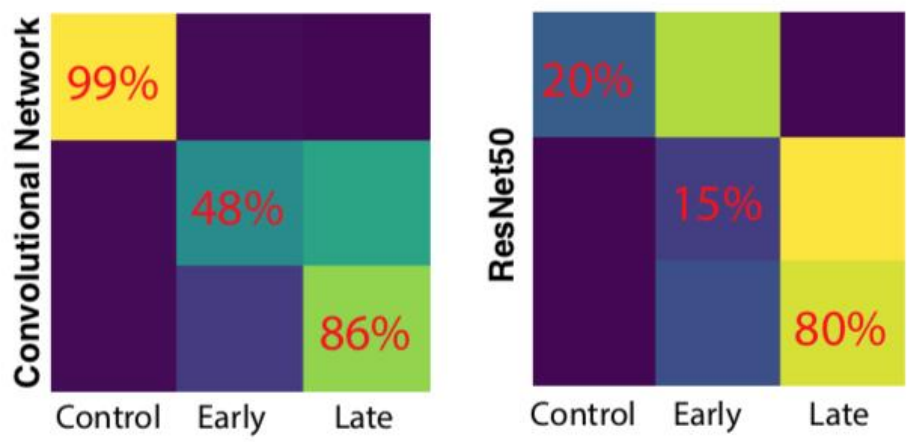

B

\begin{tabular}{|c|c|c|c|c|}
\hline & RF & CNN_1 & CNN_2 & ResNet50 \\
\hline Accuracy & $87 \%$ & $78 \%$ & $71 \%$ & $38 \%$ \\
\hline F1 score & 0.93 & 0.87 & 0.83 & 0.55 \\
\hline
\end{tabular}




\section{Supplementary Figure S9}

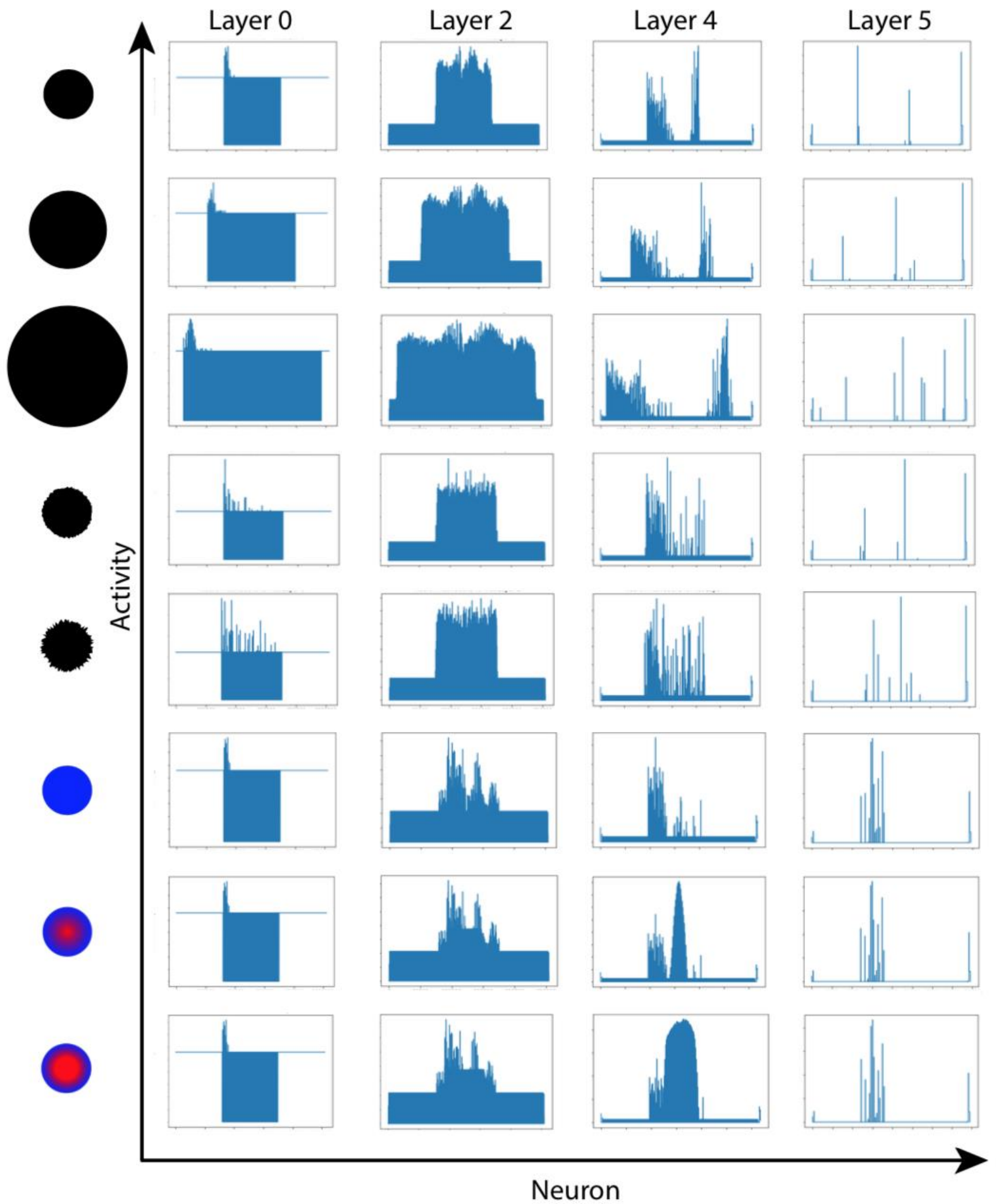


Supplementary Figure S10

A

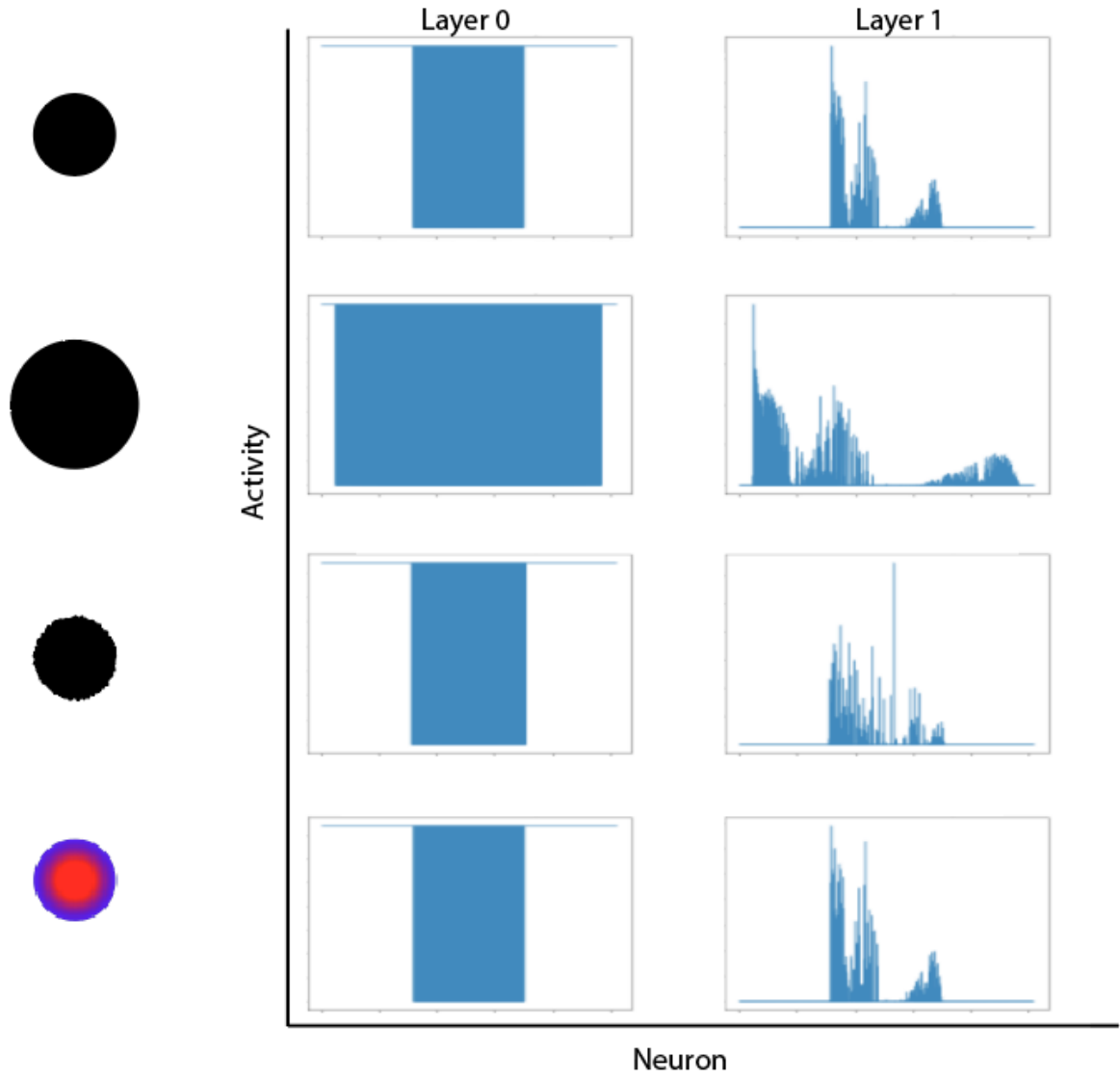

B

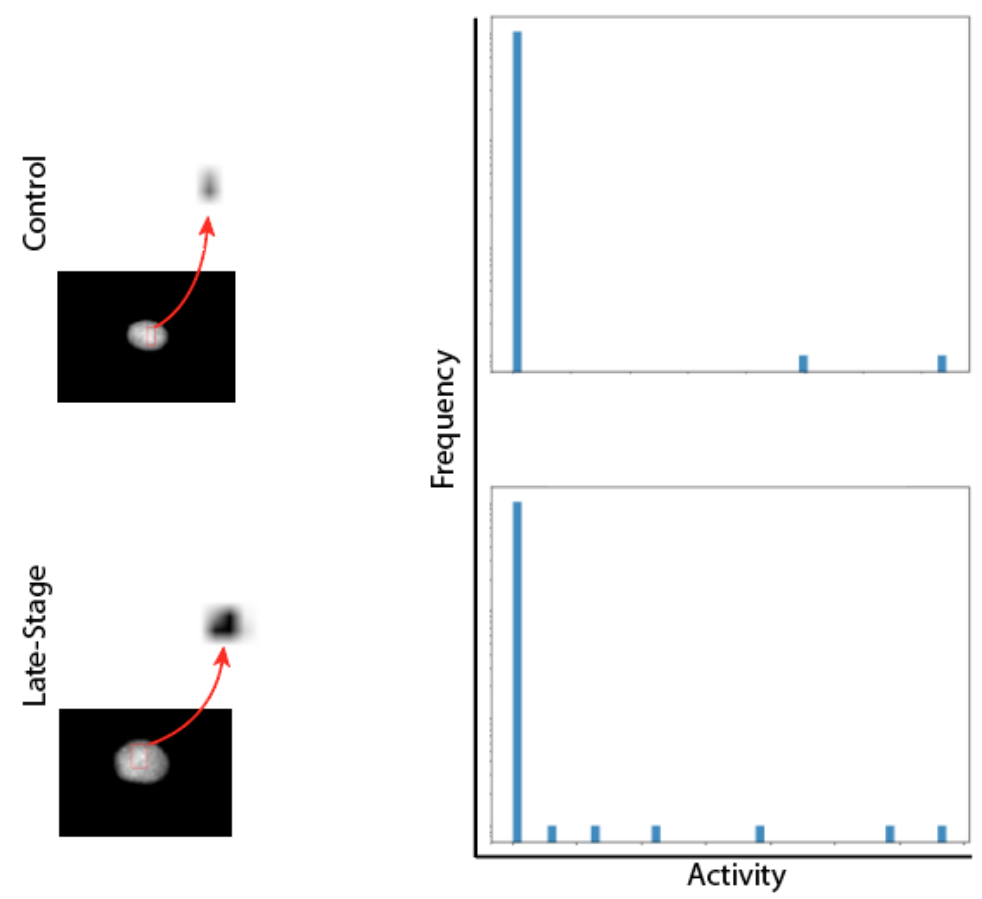




\section{References}

${ }^{1} \mathrm{He}, \mathrm{K} .$, Zhang, X., Ren, S., Sun, J. "Deep Residual Learning for Image Recognition.” Arxiv Preprint. arXiv: 1512:03385. (2015).

${ }^{2}$ Wang, A., Zhou, H., Xu, W., Chen, X. (2018). "Deep Neural Network Capacity." Arxiv Preprint. arXiv:1708.05029

${ }^{3}$ Yosinski, J., Clune, J., Bengio, Y., Lipson, H. (2014). "How transferrable are features in deep neural networks?" Advances in Neural Information Processing Systems 27, pp. 3320-3328 